\title{
On the Length of a Partial Independent Transversal in a Matroidal Latin Square
}

\author{
Daniel Kotlar \\ Department of Computer Science \\ Tel-Hai College \\ Upper Galilee 12210, Israel \\ dannykot@telhai.ac.il
}

\author{
Ran Ziv \\ Department of Computer Science \\ Tel-Hai College \\ Upper Galilee 12210, Israel \\ ranziv@telhai.ac.il
}

Submitted: Jan 13, 2012; Accepted: Apr 9, 2012; Published: Apr 16, 2012 Mathematics Subject Classifications: 68R05, 05B15, 05B35, 15A03

\begin{abstract}
We suggest and explore a matroidal version of the Brualdi - Ryser conjecture about Latin squares. We prove that any $n \times n$ matrix, whose rows and Columns are bases of a matroid, has an independent partial transversal of length $\lceil 2 n / 3\rceil$. We show that for any $n$, there exists such a matrix with a maximal independent partial transversal of length at most $n-1$.
\end{abstract}

Keywords: Latin square, matroidal Latin square, partial independent transversal

\section{Introduction}

A Latin Square of order $n$ is an $n \times n$ array $L$ with entries taken from the set $\{1, \ldots, n\}$, where each entry appears exactly once in each row or column of $L$. A partial transversal of size $k$ of a Latin square $L$ is a subset of $k$ different entries of $L$, where no two of them lie in the same row or column.

The maximal size of a partial transversal in $L$ will be denoted here by $t(L)$ and the minimal size of $t(L)$, over all Latin squares $L$ of order $n$, will be denoted by $T(n)$.

It has been conjectured by Ryser [10] that $T(n)=n$ for every odd $n$ and by Brualdi [4] (see also [2] p. 255) that $T(n)=n-1$ for every even $n$. Although these conjectures are still unsettled, a consistent progress has been made towards its resolution: Koksma [8] proved that for $n \geqslant 3, T(n) \geqslant\lceil(2 n+1) / 3\rceil$. This bound was improved by Drake [5] to $T(n) \geqslant\lceil 3 n / 4\rceil$ for $n>7$, and again by de Veris and Wieringa [3] who obtained a lower bound of $\lceil(4 n-3) / 5\rceil$ for $n \geqslant 12$. Woollbright [14] showed that $T(n) \geqslant\lceil n-\sqrt{n}\rceil$. A similar result was obtained independently by Brouwer, de Vries and Wieringa [1]. Recently, Hatami and Shor [6] proved that $T(n) \geqslant n-O\left(\log ^{2} n\right)$. See also a recent comprehensive survey by Wanless [11]. 
The aim of this note is to suggest and explore a matroidal version of the Brualdi-Ryser conjectures. For basic texts on matroids the reader is referred to Welsh [12], Oxley [9] and White [13].

Definition 1. Let $(M, S)$ be a matroid $M$ on a ground set $S$. A matroidal Latin square (abbreviated MLS) of degree $n$ over $(M, S)$ is an $n \times n$ matrix $A$ whose entries are elements of $S$, where each row or column of $A$ is a base of $M$.

Notice that a matroidal Latin square reduces to a Latin square if $\mathrm{M}$ is a partition matroid. We mention that according to a well-known conjecture of Rota [7] every set of $n$ bases of a matroid of rank $n$ can be arranged to form an MLS of degree $n$ so that its rows consist of the original bases.

Definition 2. An independent partial transversal of an MLS $A$ is an independent subset of entries of $A$ where no two of them lie in the same row or column of $A$.

We propose the following analogue of Brualdi's conjecture:

Conjecture 3. Every MLS of degree $n$ has an independent partial transversal of size $n-1$.

In view of Ryser's conjecture, it is natural to ask whether in Conjecture 3 an independent transversal of size $n$ exists whenever $n$ is odd. Theorem 6 asserts that this is not the case.

\section{A lower bound for a maximal independent partial transversal}

Let $A=\left(a_{i j}\right)_{i, j=1}^{n}$ be an MLS of degree $n$ over a matroid $M$. Let $T$ be an independent partial transversal of size $t$. Without loss of generality we may assume that the elements of $T$ are the first $t$ elements of the main diagonal of $A$. That is

$$
A=\left(\begin{array}{c|c}
B & C \\
\hline D & E
\end{array}\right)
$$

where $B, C, D$ and $E$ are sub-matrices of $A$ of dimensions $t \times t, t \times(n-t),(n-t) \times t$ and $(n-t) \times(n-t)$ respectively, and $T$ constitutes the main diagonal of $B$. If $T$ is of maximal length, then $t \geqslant\lceil n / 2\rceil$. Otherwise $\operatorname{dim}(E) \geqslant n-t>t=\operatorname{dim}(T)$ and thus $E$ would contain an element that is not spanned by $T$ and hence can be added to $T$, contradicting the maximality of $T$. In order to show that $t \geqslant\lceil 2 n / 3\rceil$ we shall need the following lemma:

Lemma 4. Let $X$ be a finite set and let $s>|X| / 2$. Let $X_{1}, \ldots, X_{s}$ be a family of subsets of $X$, each of size at least s. Then there exists some $X_{i}$, all of whose elements appear in other subsets in the family. 
Proof. Let $Y_{1}$ be the set of elements in $X$ that appear in exactly one of the subsets $X_{1}, \ldots, X_{s}$ and let $Y_{2}$ be the set of elements in $X$ that appear in at least two of the subsets $X_{1}, \ldots, X_{s}$. Let $k_{1}=\left|Y_{1}\right|$ and $k_{2}=\left|Y_{2}\right|$. Assume, by contradiction, that each $X_{i}$ contains at least one element of $Y_{1}$. Then $k_{1} \geqslant s$ and thus

$$
k_{2} \leqslant|X|-k_{1} \leqslant|X|-s<|X| / 2
$$

(since $s>|X| / 2$ ). If, for some $i,\left|X_{i} \cap Y_{1}\right|=1$ then $\left|X_{i} \cap Y_{2}\right| \geqslant s-1$ and thus $k_{2} \geqslant s-1>|X| / 2-1$. It follows that $k_{2} \geqslant|X| / 2$, contradicting (2). It follows that for all $i,\left|X_{i} \cap Y_{1}\right| \geqslant 2$. Then $k_{1} \geqslant 2 s$ and thus $k_{2} \leqslant|X|-k_{1} \leqslant|X|-2 s<|X|-|X|=0$, which is absurd. This proves the lemma.

Theorem 5. Let $A$ be an $M L S$ of degree $n$ over a matroid $M$. Then $A$ contains an independent partial transversal of size $\lceil 2 n / 3\rceil$.

Proof. We use the notations from the beginning of Section 2. Since $T$ is maximal, all the elements in the sub-matrix $E$ are spanned by $T$. Let $T_{E}$ be the minimal subset of $T$ that spans $E$ (this set is unique since $T$ is independent.) Since $\operatorname{dim}(E) \geqslant n-t$ then $\left|T_{E}\right| \geqslant n-t$ and thus $\left|T \backslash T_{E}\right| \leqslant t-(n-t)=2 t-n$. Since each row of $A$ is a base and all the elements of $E$ are spanned by $T$, each row of the sub-matrix $D$ contains a subset of size $n-t$ that complement $T$ to a base. In particular, each row of $D$ contains at least $n-t$ elements that are not spanned by $T$. Let $X=\{1, \ldots, t\}$ be the set of indices of the columns of $D$. For each of the $n-t$ rows in $D$ we define a subset $X_{i} \subseteq X$, $i=t+1, \ldots, n$, in the following way: $j \in X_{i}$ if and only if the $j$ th element of the $i$ th row of $A$ is not spanned by $T$. It follows that $\left|X_{i}\right| \geqslant n-t$ for all $i=t+1, \ldots, n$. Now assume, by contradiction, that $t<2 n / 3$. Then $n-t>n / 3>t / 2$. So we have a set $X$ of size $t$ and $n-t$ subsets $X_{t+1}, \ldots, X_{n}$, each of size at least $n-t$, such that $n-t>t / 2$. Let $s=n-t$. By Lemma 4 we conclude that there exists a subset $X_{i}$ all of whose elements are contained in other subsets in the family $X_{t+1}, \ldots, X_{n}$. This means that there is a row in $D$ containing at least $n-t$ elements that are not spanned by $T$ and for each such element there exists another element in the same column in $D$ that is not spanned by $T$. It follows that $D$ contains at least $n-t$ columns, each containing at least two elements that are not spanned by $T$. Since $t<2 n / 3$ we have that $\left|T \backslash T_{E}\right| \leqslant 2 t-n<n / 3<n-t$. So there exists $j \leqslant t$ such that (1) $a_{j j} \in T_{E}$ and (2) the $j$ th column of $D$ contains at least two elements that are not spanned in $T$. Let $x \in E$ be such that its support (i.e., its minimal spanning set) in $T$ contains $a_{j j}$ and let $y$ and $z$ be two elements in the $j$ th column of $D$ that are not spanned by $T$. We may assume that $x$ and $y$ are not in the same row (otherwise we take $z$ instead of $y$ ). Since $T \cup\{y\}$ is independent, and the support of $x$ in $T$ contains $a_{j j}$, it follows that $T \backslash\left\{a_{j j}\right\} \cup\{y\}$ does not span $x$ and thus $S \backslash\left\{a_{j j}\right\} \cup\{x, y\}$ is an independent partial transversal in $A$ of length $t+1$, contrary to the maximality of $T$. Thus $t$ must be at least $\lceil 2 n / 3\rceil$. 


\section{An upper bound of size $n-1$ for an MLS of degree $n$}

It is well known that for any even $n$ there exist Latin squares of order $n$ with no transversal of size $n$. The following theorem shows that for any $n$ there exists an MLS of degree $n$ with no independent transversal of size $n$.

Theorem 6. Let $v_{1}, v_{2}, \ldots, v_{n}$ be a basis of a vectorial matroid of rank $n$. Then the matrix $A=\left(a_{i j}\right)_{i, j=1}^{n}$, whose elements are $a_{i i}=v_{1}$, for $i=1, \ldots, n$, and $a_{i j}=v_{i}-v_{j}$, for $1 \leqslant i \neq j \leqslant n$, is an MLS of order $n$ with no independent transversal of size $n$.

Proof. We leave it to the reader to check that the rows and columns of $A$ are independent. Let $T$ be a transversal of size $n$ in $A$. We show that $T$ is not independent. If $T$ does not contain elements of the main diagonal of $A$, then, since each row and column is represented exactly once among the elements of $T$, the sum of the elements of $T$ is 0 , and $T$ is not independent. Thus we may assume that $T$ meets the main diagonal exactly once. Let $a_{i i}=v_{1} \in T$. If $i=1$ then the sum of the elements of $T-a_{11}$ is 0 . If $i>1$, then $v_{i}$ is not spanned by $T$, so $T$ is not a basis, and thus, is not independent.

\section{References}

[1] A. E. Brouwer, A. J. de Vries, and R. M. A. Wieringa. A lower bound for the length of partial transversals in a Latin square. Nieuw Arch. Wiskd., 24(3):330-332, 1978.

[2] R. A. Brualdi and H. J. Ryser. Combinatorial Matrix Theory. Cambridge University Press, 1991.

[3] A. J. de Vries and R. M. A. Wieringa. Een ondergrens voor de lengte van een partiele transversaal in een Latijns vierkant. preprint.

[4] J. Dénes and A. D. Keedwell. Latin squares and their applications. Academic Press, New York, 1974.

[5] D. A. Drake. Maximal sets of Latin squares and partial transversals. J. Statist. Plann. Inference, 1:143-149, 1977.

[6] P. Hatami and P. W. Shor. A lower bound for the length of a partial transversal in a Latin square. J. Combin. Theory A, 115:1103-1113, 2008.

[7] R. Huang and G-C. Rota. On the relations of various conjectures on Latin squares and straightening coefficients. Discrete Mathematics, 128:225-236, 1994.

[8] K. K. Koksma. A lower bound for the order of a partial transversal in a Latin square. J. Combin. Theory, 7:94-95, 1969.

[9] J. Oxley. Matroid Theory. Oxford University Press, 2 edition, 2011.

[10] H. J. Ryser. Neuere probleme der kombinatorik. In Vorträge über Kombinatorik, Oberwolfach, Matematisches Forschungsinstitute, pages 69-91, Oberwolfach, Germany, July 1967. 
[11] I. M. Wanless. Transversals in Latin squares: A survey, volume 392 of Surveys in Combinatorics, London Mathematical Society Lecture Note Series, pages 403-437. Cambridge University Press, 2011.

[12] D. Welsh. Matroid Theory. Academic Press, London, 1976.

[13] N. White, editor. Encyclopedia of Mathematics and its Applications, Theory of Matroids, volume 26. Cambridge University Press, 1986.

[14] D. E. Woolbright. An $n \times n$ Latin square has a transversal with at least $n-\sqrt{n}$ distinct elements. J. Combin. Theory A, 24:235-237, 1978. 